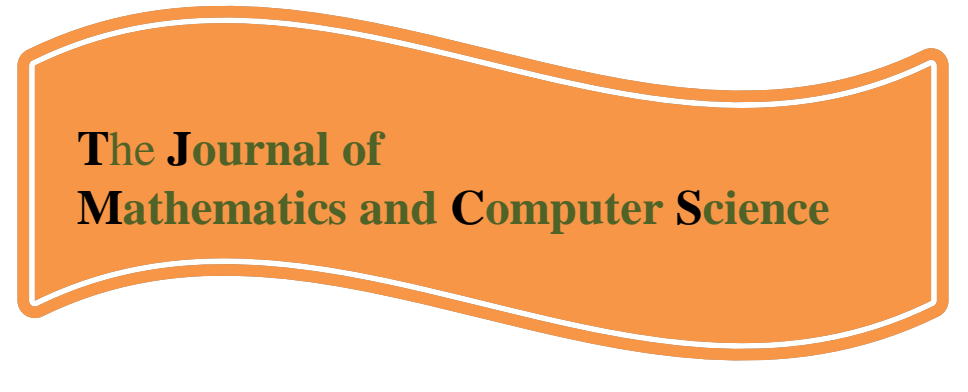

Available online at

\title{
http://www.TIMCS.com
}

The Journal of Mathematics and Computer Science Vol .5 No.4 (2012) 265-270

\section{Neighborhood Number in Graphs}

\author{
Z. Tahmasbzadehbaee ${ }^{1}$ \\ Department of Mathematics, University of Mysore, Manasagangotri, Mysore-570 006, India \\ ztahmasb@yahoo.com \\ N. D. Soner ${ }^{2}$ \\ Department of Mathematics, University of Mysore, Manasagangotri, Mysore-570 006, India \\ ndsoner@yahoo.co.in

\section{A. Mojdeh ${ }^{3}$} \\ Department of Mathematics, University of Tafresh, Tafresh, Iran \\ damojdeh@tafreshu.ac.ir
}

Received: November 2012, Revised: December 2012

Online Publication: December 2012

\section{Abstract}

A set $S$ of points in graph $G$ is a neighborhood set if $G=\bigcup_{v \in S}\langle N[v]\rangle$ where $\langle N[v]\rangle$ is the subgraph of $G$ induced by $v$ and all points adjacent to $v$. The neighborhood number, denoted $n_{o}(G)$, of $G$ is the minimum cardinality of a neighborhood set of $G$. In this paper, we study the neighborhood number of certain graphs.

Keywords: Neighborhood set; Neighborhood number; Jahangir graph; Harary graphs; Circulant graph.

\section{Mathematics Subject Classification: $05 C 69$.}

\section{Introduction}

In this paper, we concerned only with undirected simple graphs (loops and multiple edges are not allowed). All notations on graphs that are not defined here can be found in [6].

We denote the vertex set and the edge set of a graph $G$ by $V(G)$ and $E(G)$, respectively. For any vertex $v$ of $G$, the open neighborhood of $v$ is the set $N(v, G)=\{u \in V(G): u v \in E(G)\}$, while the closed neighborhood of $v$ is the set $N[v, G]=N(v, G) \cup\{v\}$. The degree of $v$ is defined as $\operatorname{deg}(v, G)=|N(v, G)|$. The maximum and minimum degree of vertices in $V(G)$ are denoted by 
$\Delta(G)$ and $\delta(G)$, respectively. If $\Delta(G)=\delta(G)=k$ then $G$ is said to be $k$-regular. We denote the distance between two vertices $x$ and $y$ in $G$ by $d_{G}(x, y)$. For a set of vertices $S \subseteq V(G), N(S, G)$ is the union of $N(x, G)$ for all $x \in S$, and $N[S, G]=N(S, G) \cup\{S\}$. A cycle on $n$ vertices is denoted by $C_{n}$.

A set $S$ of vertices in a graph $G$ is a neighborhood set (written $n_{o}$-set) of $G$, if $G=\bigcup_{v \in S}\langle N[v]\rangle$ where $\langle N[v]\rangle$ is the subgraph of $G$ induced by $v$ and all vertices adjacent to $v$. The neighborhood number $n_{o}(G)$ of $G$ is the minimum cardinality of $n_{o}$-sets of $G$. An $n_{o}$-set of $G$ with cardinality $n_{o}(G)$ is called $n_{o}$-set of $G$. We give $n_{o}$-critical if the removal of any vertex from the graph decreases the close neighborhood number. For more on concepts of domination and neighborhood critical see $[3,4,5]$.

For $m, n \geq 2$, the generalized Jahangir graph $J_{m, n}$ is a graph on $m n+1$ vertices, i.e., a graph consisting of a cycle $C_{m n}$ with one additional vertex which is adjacent to $n$ vertices of $C_{m n}$ at distance $m$ to each other on $C_{m n}$.

For $2 \leq k<n$, the Harary graph $H_{k, n}$ on $n$ vertices is defined in [9] as follows. Place $n$ vertices around a circle, equally spaced. If $k$ is even, $H_{k, n}$ is formed by making each vertex adjacent to the nearest $\frac{k}{2}$ vertices in each direction around the circle. If $k$ is odd and $n$ is even, $H_{k, n}$ is formed by making each vertex adjacent to the nearest $\frac{k-1}{2}$ vertices in each direction around the circle and to the diametrically opposite vertex. In both cases, $H_{k, n}$ is $k$-regular. If both $k$ and $n$ are odd, $H_{k, n}$ is constructed as follows. It has vertices $0,1, \ldots, n-1$ and is constructed from $H_{k-1, n}$ by adding edges joining vertex $i$ to vertex $i+\frac{n-1}{2}$ for $0 \leq i \leq \frac{n-1}{2}$.

The circulant graph $C_{n+1}\langle 1, k\rangle$ is the graph with vertex set $\left\{v_{0}, v_{1}, \ldots, v_{n}\right\}$ and edge set $\left\{v_{i} v_{i+j(\bmod n+1)} \mid i \in\{0,1, \ldots, n\}\right\}$ and $j \in\{1, k\}$ where $k \geq 2$ is an integer.

It is necessary for circulant graphs to be connected [2]. Theoretical properties of circulant graphs have been studied extensively and are surveyed [1].

Here we would like to study the neighborhood parameter of these family of graphs.

\section{Main results}

We make the following observations and proposition from [5].

Observation 2.1. [5] $n_{o}\left(C_{n}\right)=\left\lceil\frac{n}{2}\right\rceil$.

Observation 2.2. [5] $n_{o}\left(K_{n}\right)=1$.

Proposition 2.3. [5] For a graph $G$ of order $n, n_{o}(G)=1$ if and only if $G$ has a point of degree $n-1$.

The following result can be implied by Proposition 2.3. 
Lemma 2.4. $n_{o}(G)=1$ where $G \in\left\{H_{m, m+1}, H_{r, r+2}\right\}$ and $r$ is an odd integer.

Theorem 2.5. $n_{o}\left(J_{m, n}\right)=\left\{\begin{array}{l}n_{o}\left(C_{m n}\right) \quad \text { where } m \text { is even } \\ n_{o}\left(C_{m n}\right)+1 \text { where } m \text { is odd }\end{array}\right.$.

Proof. Let $\left\{x_{1}, x_{2}, \ldots, x_{m n}, u\right\}$ be the vertex set of $J_{m, n}$. We consider the following two cases:

Case 1: If $m$ is even. Define $S=\left\{x_{1}, x_{3}, \ldots, x_{m n-1}\right\}$. It is easy to check that $S$ is the neighborhood set of $J_{m, n}$ where $m$ is even. It follows that $n_{o}\left(J_{m, n}\right) \leq n_{o}\left(C_{m n}\right)$. Furthermore, $S$ is $n_{o}$-set of $C_{m n}$, too. It suffices to note that $u$ cannot be belong to any $n_{o}$-set of $J_{m, n}$ where $m$ is even. Hence, the desired result completes.

Case 2. If $m$ is odd. Define $S=D \cup\{u\}$ where $D$ is an $n_{o}$-set of $C_{m n}$. It is not so difficult to check that $u$ is belongs to any $n_{o}$-set of $J_{m, n}$ where $m$ is odd. Hence the result holds.

It is well-known that $H_{2, n}=C_{n}$. Therefore, we may assume that $m \geq 4$ in the following result.

Theorem 2.6. Let $m$ be an even integer. Then $n_{o}\left(H_{m, n}\right)=\left\lceil\frac{n}{\frac{m}{2}+1}\right\rceil$ where $n \geq m+2$.

Proof. Let $n=k\left(\frac{m}{2}+1\right)+l$ where $k \geq 2$ and $0 \leq l \leq \frac{m}{2}$. It is easy to check that $S=\left\{x_{1}\right.$, $\left.x_{1+\left(\frac{m}{2}+1\right)}, x_{1+2\left(\frac{m}{2}+1\right)}, \ldots, x_{1+(k-1)\left(\frac{m}{2}+1\right)}, x_{1+k\left(\frac{m}{2}+1\right)}\right\}$ is an $n_{o}$-set of $H_{m, n}$ which implies that $n_{o}\left(H_{m, n}\right) \leq\left\lceil\frac{n}{\frac{m}{2}+1}\right\rceil$ (Note that if $l=0$, then $1+k\left(\frac{m}{2}+1\right)$ will be modulated to $n$ ). It now suffices to complete of the proof that $n_{o}\left(H_{m, n}\right) \geq\left\lceil\frac{n}{\frac{m}{2}+1}\right\rceil$.

Without loss of generality, we can assume that $x_{i} \in S$, where $S$ is an arbitrary $n_{o}$-set. We show that the longest consecutive vertex $x_{j}$ of $x_{i}$ can be in $S$ such that $d_{C_{n}}\left(x_{i}, x_{j}\right) \leq \frac{m}{2}+1$. Suppose to the contrary, that $d_{C_{n}}\left(x_{i}, x_{j}\right) \geq \frac{m}{2}+2$. It suffices that, we verify the case $d_{C_{n}}\left(x_{i}, x_{j}\right)=\frac{m}{2}+2$. One can see that there is some edge in $H_{m, n}$ between $x_{i}$ and $x_{j}$ which cannot be found in $\left\langle N\left[x_{i}\right]\right\rangle \cup\left\langle N\left[x_{j}\right]\right\rangle$. Hence the desired result completes.

Now, we study the neighborhood number of Harary graphs of odd order. Note that, if $n \in\{m+$ $1, m+2\}$, then $n_{o}\left(H_{m, n}\right)=1$ by Lemma 2.4 . Thus, we can assume that $n \geq m+3$ in the following result.

Theorem 2.7. Let $m$ be an odd integer and let $n=2 k$ or $n=2 k+1$ for every $k$. Then $n_{o}\left(H_{m, n}\right)=\left\{\begin{array}{l}k \quad \text { where } n \text { is even and } k \text { is odd } \\ k+1 \text { where } n \text { is odd or } n \text { and } k \text { are even }\end{array}\right.$. 
Proof. Let $n=2 k$ or $n=2 k+1$. Since $m$ is odd then we have $\left\lceil\frac{n}{2}\right\rceil$ diameters in $H_{m, n}$. Moreover, by the structure of the graph, it follows that $n_{o}\left(H_{m, n}\right) \geq k$ where $n$ is even and $k$ is an odd integer and $n_{o}\left(H_{m, n}\right) \geq k+1$ where $n$ is odd or $n$ and $k$ are even.

It now suffices to complete of the proof that we assign an $n_{o}(G)$-set. Let $\left\{x_{1}, x_{3}, x_{5}, \ldots, x_{n-3}, x_{n-1}\right\}$ where $n$ is even and $k$ is odd. Let $\left\{x_{1}, x_{3}, x_{5}, \ldots, x_{n-2}, x_{n}\right\}$ where $n$ is odd and $k$ is even. Let $\left\{x_{1}, x_{3}, x_{5}, \ldots, x_{\left[\frac{n}{2}\right]-1}, x_{\left[\frac{n}{2}\right\rceil+1}, x_{\left[\frac{n}{2}\right\rceil+2}, x_{\left[\frac{n}{2}\right\rceil+4}, \ldots, x_{n-1}\right\} \quad$ where $n \quad$ and $k$ are odd. Let $\left\{x_{1}, x_{3}, \ldots, x_{\frac{n}{2}-3}, x_{\frac{n}{2}-1}, x_{\frac{n}{2}}, x_{\frac{n}{2}+2}, x_{\frac{n}{2}+4}, \ldots, x_{n-2}, x_{n-1}\right\}$ where $n$ and $k$ are even. Hence, by these assumptions, the desired result completes.

Now, we study the neighborhood number of circulant graphs. Note that, if $m=2$, then $C_{n+1}\langle 1,2\rangle=H_{4, n}$ which verified in Theorem 2.6. From this, we may assume that $m \geq 3$ in the following result.

Theorem 2.8. Let $G=C_{n+1}\langle 1, m\rangle$ where $m$ and $n$ are odd integers. Then $n_{o}(G)=n_{o}\left(C_{n+1}\right)$.

Proof. Let $G=C_{n+1}\langle 1, m\rangle$ where $m$ and $n$ are odd integers. It is clear to see that $n_{o}(G) \geq$ $n o(C n+1)$. It suffices to complete of the proof that $\{x 1, x 3, x 5, \ldots, x n\}$ is an no-set of $G$.

Assume that $C_{i}$ and $C_{j}$ are two $m+1$-cycles of circulante graph $C_{n+1}\langle 1, m\rangle$, we say these two cycles are independent, that is $V\left(C_{i}\right) \cap V\left(C_{j}\right)=\emptyset$.

Theorem 2.9. Let $G=C_{n+1}\langle 1, m\rangle$ where $m$ and $n+1$ are odd integers such that $n+1=$ $k(m+1)+l$ for every odd $l$ with $1 \leq l \leq m$. Then $n_{o}(G)=k \cdot n_{o}\left(C_{m+1}\right)+\frac{m+l}{2}$.

Proof. Let $n+1=k(m+1)+l$ for every odd $l$ with $1 \leq l \leq m$. Suppose that $\mathrm{U}_{i=1}^{k}\left\{x_{1}^{i}, x_{2}^{i}, x_{3}^{i}, \ldots\right.$ ,$\left.x_{m+1}^{i}\right\} \cup \cup_{j=1}^{l}\left\{y_{j}\right\}$ and $S$ are the vertex set and $n_{o}$-set of $C_{n+1}\langle 1, m\rangle$, respectively. It is easy to see that, we have $k$ independent $m+1$-cycle. Suppose that $C_{m+1}^{i}: x_{1}^{i}, x_{2}^{i}, \ldots, x_{m+1}^{i}$ is an $m+1$-cycle. Certainly, $\left|S \cap C_{m+1}^{i}\right| \geq n_{o}\left(C_{m+1}\right)$ for each $1 \leq i \leq k$. It follows that $n_{o}\left(C_{n+1}\langle 1, m\rangle\right) \geq k$. $n_{o}\left(C_{m+1}\right)$. Now, let $D \subseteq S$, for which $S$ and $D$ are $n_{o}$-set of $G$ and $k$ independent $m+1$-cycle in $\mathrm{H}=\mathrm{U}_{v \in D}\langle N[v]\rangle$, respectively. It is not difficult to see that, we have $m+1$ vertices in $H$ such that $m-1$ vertices of degree $3, l-1$ vertices of degree 1 and two vertices of degree 2 . By these assumptions, it follows that $n_{o}\left(C_{n+1}\langle 1, m\rangle\right) \geq k \cdot n_{o}\left(C_{m+1}\right)+\frac{m+l}{2}$ where $m$ and $n+1$ are odd integers.

It suffices to complete of the proof that, $S=\mathrm{U}_{i=1}^{k}\left\{x_{1}^{i}, x_{3}^{i}, \ldots, x_{m}^{i}\right\} \cup\left\{x_{m+1}^{k}, x_{m-1}^{k}, x_{m-3}^{k}, \ldots, x_{m-t}^{k}\right\} \cup$ $\mathrm{U}_{j=1}^{l}\left\{y_{j}\right\}$ is an $n_{\mathrm{o}}$-set of graph for which $l$ is an odd integer, $1 \leq l<m$ and $t=m-l-3$. Finally, let $S=\mathrm{U}_{i=1}^{k}\left\{x_{1}^{i}, x_{3}^{i}, \ldots, x_{m}^{i}\right\} \cup \cup_{j=1}^{l}\left\{y_{j}\right\}$ where $l=m$.

Theorem 2.10. Let $G=C_{n+1}\langle 1, m\rangle$ where $m$ is an even integer. Then 
Z. Tahmasbzadehbaee, N. S. Soner, D. A. Mojdeh / TJMCS Vol .5 No.4 (2012) 265-270

$n_{\circ}(G)=\left\{\begin{array}{lr}k \cdot n_{\circ}\left(C_{m+1}\right)+l & \text { for every even } l \leq m-2 \\ k \cdot n_{\circ}\left(C_{m+1}\right)+l-1 & \text { for } l=m \\ k \cdot n_{\circ}\left(C_{m+1}\right)+\left[\frac{m-1}{2}\right] & \text { for every odd } l \leq m-3^{*} \\ k \cdot n_{\circ}\left(C_{m+1}\right)+\frac{m}{2} & \text { for } l=m-1\end{array}\right.$

Proof. Let $n+1=k(m+1)+l$ where $0 \leq l \leq m$. Suppose that $\cup_{i=1}^{k}\left\{x_{1}^{i}, x_{2}^{i}, x_{3}^{i}, \ldots, x_{m+1}^{i}\right\} \cup$ $\cup_{j=1}^{l}\left\{y_{j}\right\}$ and $S$ are the vertex set and $n_{o}$-set of $C_{n+1}\langle 1, m\rangle$, respectively. (note that if $l=0$, then $Y=\mathrm{U}_{j=1}^{I}\left\{y_{j}\right\}=\emptyset$ ). It is easy to see that we have $k$ independent $m+1$-cycle. Suppose that $C_{m+1}^{i}: x_{1}^{i}, x_{2}^{i}, \ldots, x_{m+1}^{i}$ is an $m+1$-cycle. Certainly, $\left|S \cap C_{m+1}^{i}\right| \geq n_{o}\left(C_{m+1}\right)$ for each $1 \leq i \leq k$. It follows that $n_{o}\left(C_{n+1}(1, m)\right) \geq k \cdot n_{o}\left(C_{m+1}\right)$. Now, let $D \subseteq S$, for which $S$ and $D$ are $n_{o}$-set of $G$ and $k$ independent $m+1$-cycle in $\mathrm{H}=\mathrm{U}_{v \in D}\langle N[v]\rangle$, respectively. It is not difficult to see that:

(i) For every even $l \leq m-2$, we have $2 l+1$ vertices in $H$ such that $l+1$ vertices of degree 3 , $l-1$ vertices of degree 1 and one vertex of degree 2 . By these assumptions, it follows that $n_{0}(G) \geq k \cdot n_{0}\left(C_{m+1}\right)+l$.

(ii) For $l=m$, we have $2 l-1$ vertices in $H$ such that $l-2$ vertices of degree $3, l-2$ vertices of degree 1 and three vertices of degree 2. By these assumptions, it follows that $n_{o}(G) \geq k \cdot n_{o}\left(C_{m+1}\right)+l-1$ where $l=m$.

(iii) For every odd $l \leq m-3$, there exist $\frac{l-1}{2}$ vertices in $V(G)$ such that these do not belong to $V(H)$. Further all of them must be in $n_{\circ}(G)$-set. Also, we have $m+l-2-\frac{l-1}{2}=m-1+\frac{l-1}{2}$ vertices in $H$ such that $m-2$ vertices of degree $3, \frac{l-1}{2}+1$ vertices of degree 2 and each of these vertices, except two of them, are between a pair of those $\frac{l-1}{2}$ vertices which are not in $H$. Hence, $\frac{l-1}{2}$ vertices out of $H$ can cover at most $2 l-2$ vertices which are not of degree 4 in $H$. Meanwhile, we have $m+l-2-4\left(\frac{l-1}{2}\right)=m-l$. By these assumptions, it follows that $n_{\circ}(G) \geq$ $k \cdot n_{0}\left(C_{m+1}\right)+\left[\frac{m-1}{2}\right]$.

(iv) For $l=m-1$, there exist $\frac{l-1}{2}$ vertices in $V(G)$ such that these do not belong to $V(H)$. Further all of them must be in $n_{\circ}(G)$-set. Also, we have $m+l-1-\frac{l-1}{2}=m+\frac{l-1}{2}$ vertices in $H$ such that $m$ vertices of degree $3, \frac{l-1}{2}$ vertices of degree 2 and each of these vertices, except two of them, are between a pair of those $\frac{l-1}{2}$ vertices which are not in $H$. Hence, $\frac{l-1}{2}$ vertices out of $H$ can cover at most $2 l-2$ vertices which are not of degree 4 in $H$. Meanwhile, we have $m+l-1-4\left(\frac{l-1}{2}\right)=$ $m-l+1=2$. By these assumptions, it follows that $n_{o}(G) \geq k \cdot n_{o}\left(C_{m+1}\right)+\frac{l-1}{2}+1=k \cdot n_{o}\left(C_{m+1}\right)+$ $\frac{m}{2}$

It suffices to complete of each part of the proof that, we assign an $n_{o}$-set for the graph: 
(i) For every even $l<m$, consider $S=\mathrm{U}_{i=1}^{k}\left\{x_{1}^{i}, x_{3}^{i}, \ldots, x_{m}^{i}\right\} \cup\left\{x_{m+1}^{k}\right\} \cup Y-\left\{y_{l}\right\}$.

(ii) For $l=m$, consider $S=\cup_{i=1}^{k}\left\{x_{1}^{i}, x_{3}^{i}, \ldots, x_{m}^{i}\right\} \cup Y-\left\{y_{l-1}\right\}$.

(iii) For every odd $l \leq m-3$, let $t=m-l-3$. If $t=0$, consider $S=\mathrm{U}_{i=1}^{k}\left\{x_{1}^{i}, x_{3}^{i}, \ldots, x_{m}^{i}\right\} \mathrm{U}$ $\left\{y_{1}, y_{3}, x_{5}, \ldots, y_{l}\right\}$ and for $t \geq 1$, consider $S=\cup_{i=1}^{k}\left\{x_{1}^{i}, x_{3}^{i}, \ldots, x_{m}^{i}\right\} \cup\left\{x_{m-2}^{k}, x_{m-4}^{k}, \ldots, x_{m-t}^{k}\right\} \cup\left\{y_{1}\right.$, $\left.y_{3}, y_{5}, \ldots, y_{l}\right\}$.

(iv) For $l=m-1$, consider $S=\mathrm{U}_{i=1}^{k}\left\{x_{1}^{i}, x_{3}^{i}, \ldots, x_{m}^{i}\right\} \cup\left\{y_{1}, y_{3}, \ldots, y_{l}\right\}$.

\section{Acknowledgement}

1,2The Research work is supported by UGC-SAP-DRSI NO: F.510/2/DRS/2011 (SAP-I).

\section{References.}

[1] J. C. Bermond, F. Comellas and D. F. Hsu, Distributed loop computer networks: a survey, J. Paralled Distrib. Comput. 24 (1995), 2-10.

[2] F. Boesch and R. Tindell, Circulants and their connectivity, J. Graph Theory 8 (1984), 487-499.

[3] S. Ghobadi, N. D. Soner and D. A. Mojdeh, Vertex neighborhood critical graphs, International Journal of Mathematics and Analysis, 9(1-6)(2008), 89-95.

[4] E. Sampathkumar and P. S. Neeralagi, Independent, Perfect and Connected neighborhood number of a graph, Journal of Combinatorics, Information \& System Sciences, 19(3-4)(1994), 139-145.

[5] E. Sampathkumar and P. S. Neeralagi, The neighborhood number of a graph, Indian J. Pure Appl. Math., 16(2)(1985), 126-132.

[6] D. B. West, Introduction to graph theory (Second Edition), Prentice Hall, USA, 2001. 